$\Rightarrow 2 \mathrm{D} I \mathrm{CE}$

\section{Edgy imaging}

Water is the most common and most controversial liquid on Earth. Despite

This is the first its essential role in all aspects of life, attempts to fully understand many of its fundamental properties and physicochemical behaviours still keep many scientists busy. For example, when water freezes on a surface, it produces $2 \mathrm{D}$ ice with implications in atmospheric and planetary science, biology and tribology. An atomistic understanding of the mechanism of formation of $2 \mathrm{D}$ ice is therefore essential - but unfortunately missing - to control ice formation on surfaces. Now, writing in Nature, Ying Jiang and co-workers propose a growth mechanism for 2D ice based on direct, atomistic imaging of the ice edge structure.

The formation of low-dimensional ice is a known phenomenon, but the short life and fragility of the intermediate structures have made direct experimental observation extremely difficult. There are some structural features upon which the scientific community agree: the basal plane of bulk ice exhibits a hexagonal lattice that terminates with zig-zag rather than armchair edges as a result of a lower density of unsaturated hydrogen bonds in the former case. However, a new $\mathrm{H}$-sensitive and non-invasive atomic force microscopy (AFM) technique developed by Jiang and colleagues revealed that,

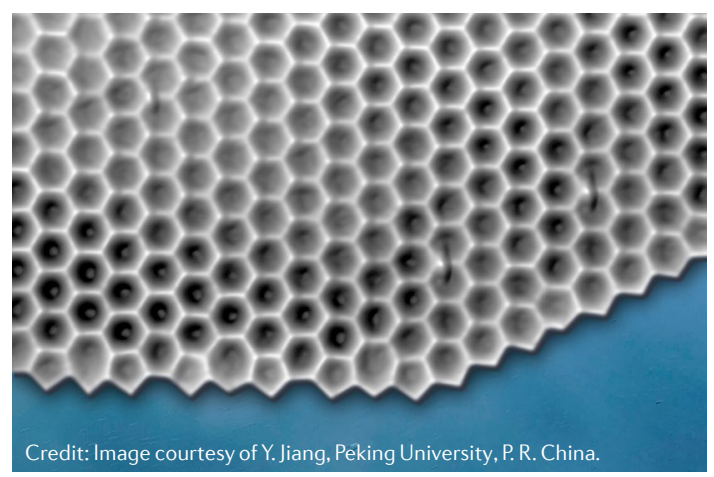

in the $2 \mathrm{D}$ regime, the edges might grow differently.

The AFM technique used in this study makes use of a qPlus sensor and a CO-functionalized tip. Functionalized AFM tips have shown great single-atom resolution for the study of organic molecules. This resolution is usually achieved by bringing the functionalized tip very close (within the Pauli repulsive regime) to the molecule under investigation - a tip-molecule separation that would likely perturb the weak hydrogen bonds between the water molecules. However, a careful control of the charge distribution at the tip apex and the tip-ice distance enabled Jiang and colleagues to tune their interactions in the force regime between van der Waals attraction and Pauli repulsion, preserving the ice structure and achieving high atomistic resolution. "Such a technique yields unprecedented submolecular-resolution images of water molecules, in which $\mathrm{H}$ and $\mathrm{O}$ atoms are distinguishable. Meanwhile, the disturbance of the CO-tip on the water can be reduced to a minimal level, thanks to the ultrahigh flexibility of the tip apex and the weak high-order electrostatic force between the tip and the water molecules. The weakly perturbative nature of the probe allows us to resolve highly fragile ice edge structures in our experiments," explains Jiang.

AFM images were collected after deeply freezing at $5 \mathrm{~K}$ several intermediate edge structures during ice growth on a $\mathrm{Au}(111)$ surface. The snapshots revealed that, in the case of $2 \mathrm{D}$ ice, armchair edges can also be stabilized after a proper rearrangement of water molecules. Whereas the zig-zag edges grow by preserving their structure, the armchair edges grow by undergoing a rearrangement of the water molecules that leads to a periodic sequence of pentagon-heptagonpentagon-hexagon (5756 structure), in agreement with molecular dynamics (MD) simulations. This molecular rearrangement minimizes the number of unsaturated $\mathrm{H}$ bonds without introducing much strain, therefore increasing the structural stability. The $2 \mathrm{D}$ ice is observed to form a bilayer and by doing so exhibits a fully saturated $\mathrm{H}$-bond structure, meaning that it can exist as a standalone crystal, also in absence of a substrate.

"Such a 2D bilayer hexagonal ice was first predicted by Koga and colleagues using MD simulations, but direct imaging of its atomic structure has been lacking until now. This is the first experiment to yield the atomic-scale information of ice growth in real space, which may change our conventional understanding of the formation and melting of low-dimensional ice at surfaces or under confinement," remarks Jiang. Furthermore, the technique and methods presented in this study might also be used to gain further understanding on the structure and dynamics of $2 \mathrm{D}$ materials other than $2 \mathrm{D}$ ices.

About the future steps, Jiang reveals "We are interested in studying the evolution from $2 \mathrm{D}$ to $3 \mathrm{D}$ ices, which has wider relevance to ice formation and growth in general. We expect to find that the $2 \mathrm{D}$ growth mode can persist up to a certain thickness, after which several ice layers will undergo a structural transformation from stacks of flat bilayer ice to interconnected buckled bilayer ice, similar to the transformation from the graphite to diamond."

Gabriella Graziano

ORIGINAL ARTICLE Ma, R. et al. Atomic imaging of the edge structure and growth of a twodimensional hexagonal ice. Nature 577, 60-63 (2020) RELATED ARTICLE Koga, K., Zeng, X. C. \& Tanaka, H. Freezing of confined water: a bilayer ice phase in hydrophobic nanopores. Phys. Rev. Lett. 79, 5262 (1997) 